\title{
Diffusion Behaviour of Methane in Different Kerogen Matrices
}

\author{
KAI BIN YU AND OZGUR YAZAYDIN
}

University College London

Presenting Author: kaibin.yu@ucl.ac.uk

Access to energy is at the heart of societal development, energy scarcity has prompted us to resort to alternative resources. Shale gas recovered from source rocks as an energy resource, though unconventional, is attracting considerable attention due to its untapped potentials. Recent development in shale extraction techniques has made the recovery process becoming more lucrative and economically viable. Kerogen is thought to play an important role in gas recovery due to being the primary organic component of shale. However, the interactions between trapped shale gas and kerogen remain poorly understood due to confinement effects resulting from the latter being a heterogeneous nanoporous structure with complex pore characteristics. This study set out to examine the diffusive behaviour of methane molecules in kerogen matrices of different types (Type I, II, and II) and maturity levels ranging from A to D for Type II kerogens on a molecular scale. We employed Grand Canonical Monte Carlo (GCMC) simulations to compute the methane adsorption and then followed with equilibrium molecular dynamics (EMD) simulations to compute the mean square displacement (MSD) of methane within the kerogen matrices at reservoir-relevant conditions, i.e., $375 \mathrm{~K}$ and 275 bar. Moreover, transport diffusivity of methane was calculated through non-equilibrium molecular dynamics (NEMD) simulations. Our results have shown that self-diffusivity of methane exhibit some degree of anisotropy in all kerogen types, except for Type I-A kerogen, where isotropic diffusion can be observed. Self-diffusivity appears to correlate positively with pore volume for Type II kerogens, where an increase can be observed with maturity. Highest diffusion rate can be observed in Type I-A kerogen. Swelling upon methane adsorption can also be appreciated. The findings can contribute to a better understanding of the mechanisms of gas transport in shale and may lead to further development of extraction techniques, fracturing fluids, and recovery predictions. 